\title{
Altered Maternal Serum Dynamic Thiol-Disulfide Interchange Reactions in Pregnant Women with Gestational Diabetes Mellitus
}

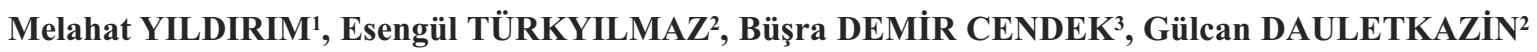 \\ Murat ALIŞIK ${ }^{4}$, Pervin BARAN ${ }^{4}$, Ayşegül CINKAYA², Ayşe Filiz AVŞAR YAVUZ1
}

Ankara, Turkey

\begin{abstract}
OBJECTIVE: We aimed to evaluate the alteration of dynamic thiol/disulfide homeostasis which basically shows the oxidative stress in body in pregnant women with gestational diabetes mellitus (GDM) by using a newly developed and automated analysis method.
\end{abstract}

STUDY DESIGN: Study population consisted of body mass index and gestational age-matched pregnant women. Patients were subdivided into 3 groups based on their response to glucose challenge test (GCT) and oral glucose tolerance test (OGTT) results: (1) control group (normal GCT test results $n=87$ ); (2) Impaired glucose tolerance (IGT) group ( $n=37$ ); (3) GDM group ( $n=25)$. Maternal serum thiol and disulphide levels were compared among three groups.

RESULTS: Maternal serum thiol values were found decreased and maternal serum disulphide levels were increased in GDM groups were compared to control and IGT groups (Thiols for controls: 398.19 $\pm 30.49 \mu \mathrm{mol} / \mathrm{L}$, for IGT group 395.92 $\pm 35.52 \mu \mathrm{mol} / \mathrm{L}$, and for GDM group 371.89 $\pm 41.14 \mu \mathrm{mol} / \mathrm{L} p=$ 0.002). Disulphides for controls; $17.47 \pm 4.38 \mu \mathrm{mol} / \mathrm{L}$, for IGT; $19.27 \pm 3.34 \mu \mathrm{mol} / \mathrm{L}$, for GDM group; $25.46 \pm 4.21 \mu \mathrm{mol} / \mathrm{L},(p=0.001)$. Disulphide/thiol ratio was found to be increased in GDM group comparing to other groups (Disulphide/thiol ratio: $0.044 \pm 0.012$ for controls, $0.049 \pm 0.009$ for IGT group, $0.068 \pm 0.0103$ for GDM group $p=0.001$ )

CONCLUSION: Thiol- disulphide balance has shifted to the oxidative side in pregnant women with GDM. So blood glucose regulation is extremely crucial for reducing the oxidative stress which may lead to damages to vital organs of a mother or possibly to development of a fetus in women with GDM.

Keywords: Gestational diabetes mellitus, Oxidative stress, Thiol/disulphide exchange, Antioxidants

Gynecol Obstet Reprod Med 2016;22(3):129-134 DOI: 10.21613/GORM.2016.71

\section{Introduction}

Gestational diabetes mellitus is defined as carbohydrate intolerance that occurs or is first recognized during pregnancy (1). The prevalence of GDM may vary from $1 \%$ to $14 \%$ of all pregnancies, depending on the population and diagnostic cri-

\footnotetext{
${ }^{I}$ Yıldırım Beyazıt University Department of Obstetrics and Gynecology Bilkent, Ankara

${ }^{2}$ Atatürk Training and Research Hospital Department of Obstetrics and Gynecology, Bilkent, Ankara

3 Etlik Zübeyde Hanım Women's Health and Education Hospital Department of Obstetrics and Gynecology, Ankara

${ }^{4}$ Atatürk Training and Research Hospital Department of Biochemistry Bilkent, Ankara

Address of Correspondence: Melahat Yıldırım Ylldırım Beyazıt University Department of Obstetrics and Gynecology, Bilkent Ankara, Turkey melahatyildrim@yahoo.com

Submitted for Publication: 07. 04.2016

Accepted for Publication:

20. 04.2016
}

teria (2); The incidence of the disease is tend to increase with advancing maternal age and obesity (3). Decreased insulin sensitivity, inadequate insulin secretion from the pancreatic beta cells, and hyperglycemia are the constant components of this heterogeneous disorder (3). GDM is found to be related to increased risks of macrosomia, shoulder dystocia, birth injuries, preeclampsia, increased risk of developing type 2 diabetes in mothers and long term health problems with respect to childhood obesity in offspring (4).

Oxidative stress is defined as a perturbation in the balance between the production of reactive oxygen species (ROS) and antioxidant defenses leading to tissue damages in organisms (5). Oxidative stress has been found to be connected to the pathogenesis of numerous diseases including atherosclerosis, diabetes, hypertension, and cancers $(6,7)$. The balance between ROS and antioxidants is crucial to maintain physiological functions of cells, tissues, and the whole body. The mechanisms that how oxidative stress affects the vital organs are well described (3), moreover, the association between diabetes especially type 2 diabetes mellitus (DM) and oxidative stress 
has been well documented in literature $(8,9)$ identifying increased ROS and impaired antioxidant defense system in type 2 diabetic patients. However, only limited number of studies have been conducted to date the association between oxidative stress and the pathogenesis of GDM which is similar to type 2 DM $(10,11)$.

Thiol/disulfide exchange reactions are well-known protective mechanisms against the oxidative damage in the body (12). Alteration in redox reactions involving thiols and disulfides also contribute to cellular protection against oxidation. Thiols are organic compounds with carbon-bonded sulfhydryl and hydrogen groups. In presence of oxidative stress, thiols react with oxidizing agents and mediate the formation of reversible disulphide bonds between proteins. Disulphides mainly found in proteins located outside the cells forming bridge-like structures can function to regulate the response to oxidative challenge (12). If thiol/disulphide homeostasis switches in favor of disulphides, it may lead to increased oxidative response in the body. Increased thiol levels, on the other hand, provide substantial antioxidant protection against ROS, and support cellular signaling mechanisms, and regulation of vital enzymes $(12,13)$. Although one part of this twosided balance determined in 1979, a newly developed automated analysis method by Erel allowed the measurements of serum thiols and disulphides either separate or combined (14). In this convenient method, serum thiol concentrations can be measured and plasma thiol/disulphide homeostasis can be determined easily.

In this study we aimed to evaluate the alteration of dynamic thiol/disulfide homeostasis in pregnant women with gestational diabetes by using a new and automated analysis method developed by Erel (14).

\section{Material and Method}

This prospective observational study was carried out at the Obstetrics and Gynecology Clinic of Ankara Ataturk Training and Research Hospital between January 2015 and December 2015 . The study protocol was approved by the ethics committee of the same intuition and complied with the Helsinki Declaration. A written informed consent was obtained from all subjects in the study. A total of 146 pregnant women with singleton pregnancies were included in the study. Gestational age was determined based on the combination of the last menstrual period and ultrasound findings in the first trimester. Body mass index (BMI) was calculated as weight $(\mathrm{kg}) /$ height squared $\left(\mathrm{m}^{2}\right)$ in all cases. Patient characteristics (age, gravida , parity, pregestational BMI and historical information were collected from subjects. All participants underwent the obstetrical evaluation and received an oral glucose challenge test (GCT) between 24-28 weeks of gestation. During this period, all women were advised to admit to antenatal clinics with a fasting state. Before GCT, participants were kindly asked to give blood samples for the evaluation of fasting blood glucose, glycosylated hemoglobin A1c (HbA1c), fasting insulin, and serum thiol and disulphide levels. Then $50 \mathrm{~g}$ glucose solution was ingested by all pregnant women in order to screen GDM. For GCT, venous blood samples were obtained from the antecubital vein one hour after the ingestion of a solution. Women with a GCT $\geq 140 \mathrm{mg} / \mathrm{dl}$ were accepted as having a positive test and referred to oral glucose tolerance test (OGTT). The 100-gram OGTT was performed in the morning following overnight fasting. Venous blood samples were drawn at baseline (fasting) and 60, 120, and $180 \mathrm{~min}$ following ingestion of the standard 100-gram glucose load. OGTT results were evaluated using the Carpenter and Coustan modification of the National Diabetes Data Group (NDDG) criteria (15), which is accepted as positive OGTT as venous blood glucose levels $\geq 95 \mathrm{mg} / \mathrm{dL}$ for fasting, $\geq 180 \mathrm{mg} / \mathrm{dL}$ for $1 \mathrm{~h}, \geq$ $155 \mathrm{mg} / \mathrm{dL}$ for $2 \mathrm{~h}$, and $\geq 140 \mathrm{mg} / \mathrm{dL}$ for $3 \mathrm{~h}$. Elevation in two or more OGTT parameters at or above aforementioned levels were accepted as having GDM. Patients with GCT values at or above $200 \mathrm{mg} / \mathrm{dL}$ were considered as having GDM as well. Patients having one abnormal OGTT parameter or normal OGTT following positive GCT were defined as having impaired glucose tolerance (IGT). Patients were subdivided into 3 groups based on their response to GCT and OGTT results: (1) control group (normal GCT test results $n=87$ ); (2) IGT group ( $n=37)$; (3) GDM group $(n=25)$. Since the obesity may enhance the oxidative stress (16), all three groups were consisted of BMI and gestational weeks matched pregnant women. Women with $<18$ years old, multiple pregnancies, any infectious or inflammatory disease, preeclampsia/eclampsia, hypertension, smoking habit, alcohol consumption were excluded from the study.

Serum glucose levels were measured using the hexokinase method (COBAS Integra 800, Roche, Germany). Blood samples were collected in EDTA-contained tubes and processed in a Sysmex XE 2100 device (Roche Diagnostics, Basel, Switzerland) for complete blood count analysis. Complete blood cell count parameters (white blood cell count (WBC), hemoglobin $(\mathrm{Hgb})$, hematocrit $(\mathrm{Htc})$ of the patients were measured and recorded with the assistance of a computer.

Insulin (IU/mL) was measured with cobas e 601 (RocheHitachi Diagnostics, Mannheim, Germany ) immunoassay device by using the chemiluminescence immunoassay method.

HbA1c was measured with turbidimetric inhibition immunoassay (Cobas 8000; Roche-Hitachi Diagnostics, Mannheim, Germany) (minimum detection rate $0.1 \mathrm{~g} / \mathrm{dL}$ ). For measurement of fasting maternal serum thiol and disulphide levels, collected venous blood samples from pregnant women between 24-28 weeks of gestation were centrifugated at $2300 \mathrm{~g}$ for 10 minutes. Then serum was separated and stored at $-80^{\circ} \mathrm{C}$ until analysis. Serum thiol-disulphide homeostasis was determined with an automatic measurement method (12) by using 
an automated clinical chemistry analyzer (Roche, Cobas 501, Mannheim, Germany). Brief explanation for the process:(1) the reducible disulfide bonds were reduced to form free functional thiol groups. (2) Formaldehyde helped remove unused and consumed sodium borohydride, (3) all thiol groups, including both reduced and native groups were determined after the reaction with DTNB (5,50-dithiobis-(2-nitrobenzoic acid. Following the calculation of thiols, disulfide amounts, the percentage of disulfide/total thiol ratios were determined. Total thiol, and disulphide levels were measured amongst three groups.

The statistical analyses were conducted using IBM SPSS Statistics for Windows, version 21.0 (released 2012, IBM Corp., Armonk, N.Y., USA). The Shapiro-Wilk test was performed to test the normality of distribution of the variables. Continuous variables with normal distribution were presented as mean $\pm \mathrm{SD}$. Non-normally distributed variables were presented as median (interquartile range). Since the data were normally distributed, thiol and disulphide levels among the 3 groups were assessed by using ANOVA and post hoc Bonferroni's multiple $t$ test. Comparison of blood glucose values at baseline (fasting) and 60, 120, and 180 min between IGT and GDM groups were made by using student $\mathrm{T}$ test. Pearson test was performed to detect correlations between thiols and GCT and between disulphides and GCT values. A p value $<0.05$ was considered statistically significant

\section{Results}

Descriptive statistics and some of blood test parameters of groups are shown in table 1 . The mean age was $24.8 \pm 6.5$ years in control group, 27.6 \pm 4.7 years in IGT and 26.6 \pm 3.2 years in GDM groups $(\mathrm{p}=0.037)$ (Table 1). Pregestational BMI was found $25.24 \pm 5.02 \mathrm{~kg} / \mathrm{m}^{2}$ in control group, $26.33 \pm 4.32 \mathrm{~kg} / \mathrm{m}^{2}$ in IGT group and $26.79 \pm 5.23 \mathrm{~kg} / \mathrm{m}^{2}$ in GDM group $(\mathrm{p}=0.063)$
(Table 1). The mean gestational age was not different among three groups as $25.26 \pm 1.01$ weeks for control, $25.93 \pm 1.12$ weeks for IGT and $25.31 \pm 1.13$ weeks for GDM groups $(\mathrm{p}=0.241)$. No significant differences of $\mathrm{WBC}$ values among three groups were observed in the study $(p=0.117)$ (Table 1$)$. $\mathrm{Hgb}$ and Htc values were found significantly different among three groups $(\mathrm{p}=0.033, \mathrm{p}=0.002$ respectively) (Table 1$)$.

OGTT parameters for GDM groups were found to be higher compared with IGT group in the study (FPG: 89.11 \pm 8.9 $\mathrm{mg} / \mathrm{dL}$ vs. $80.20 \pm 7.4 \mathrm{mg} / \mathrm{dL} \mathrm{p}=0.022$, glucose at 60 minute $=$ $172.78 \pm 20.79 \mathrm{mg} / \mathrm{dL}$ vs. $147.42 \pm 27.27 \mathrm{mg} / \mathrm{dL} \mathrm{p}=0.001$, glucose at 120 minute: $149.50 \pm 14.6 \mathrm{mg} / \mathrm{dL}$ vs. $124.66 \pm 22.2$ $\mathrm{mg} / \mathrm{dL} \mathrm{p}=0.001$, glucose at 180 minute: $130.67 \pm 20.5 \mathrm{mg} / \mathrm{dL}$ vs. $113.29 \pm 22.4 \mathrm{mg} / \mathrm{dL}$ p=0.007). Fifty g GCT levels were significantly higher in the GDM group comparing to controls and IGT group ( $\mathrm{p}=0.001) . \mathrm{HgA} 1 \mathrm{c}$ levels were $4.92 \pm 0.47 \mathrm{~g} / \mathrm{dL}$ in control group, $5.25 \pm 0.44 \mathrm{~g} / \mathrm{dL}$ in IGT and $5.74 \pm 0.52 \mathrm{~g} / \mathrm{dL}$ in GDM group $(\mathrm{p}=0.001)$ (Table 2). Serum thiol levels were $398.19 \pm 30.49 \mu \mathrm{mol} / \mathrm{L}$ in controls, $395.92 \pm 35.52 \mu \mathrm{mol} / \mathrm{L}$ in IGT and $371.89 \pm 41.14 \mu \mathrm{mol} / \mathrm{L}$ in GDM group ( $\mathrm{p}=0.002)$. Post hoc Bonferroni's multiple $t$ test showed that significant differences were observed between control and IGT groups $(\mathrm{p}=0.001)$ and between IGT and GDM groups $(\mathrm{p}=0.015)$. Maternal serum disulphide values were $17.47 \pm 4.38 \mu \mathrm{mol} / \mathrm{L}$ in control group, $19.27 \pm 3.34 \mu \mathrm{mol} / \mathrm{L}$ in IGT and $25.46 \pm 4.21$ $\mu \mathrm{mol} / \mathrm{L}$ in GDM groups $(\mathrm{p}=0.001)$. Post hoc Bonferroni's multiple $t$ test demonstrated that there was a significant difference of disulphide values between control and GDM $(p=0.001)$ and between IGT and GDM groups ( $\mathrm{p}=0.001)$.

Regardless of the groups, GCT values were negatively correlated with serum thiol levels $(\mathrm{p}=0.001)$ (Figure 1) and positively correlated with serum disulphide values $(p=0.001)$ (Figure 2) in the study.

Table 1: Descriptive statistics and blood test parameters of groups

\begin{tabular}{|c|c|c|c|c|}
\hline & Control $(n=87)$ & IGT (n=37) & GDM (n=25) & $p$ \\
\hline Age (year) & $24.8 \pm 6.5$ & $27.6 \pm 4.7$ & $26.6 \pm 3.2$ & 0.037 \\
\hline Gravida (n) & $2 \pm 0.9$ & $2.24 \pm 1.1$ & $2.1 \pm 1.07$ & 0.739 \\
\hline Parity (n) & $0.81 \pm .82$ & $0.91 \pm .84$ & $0.80 \pm 0.71$ & 0.987 \\
\hline Pregestational BMI $\left(\mathrm{kg} / \mathrm{m}^{2}\right)$ & $25.24 \pm 5.02$ & $26.33 \pm 4.32$ & $26.79 \pm 5.23$ & 0.063 \\
\hline Gestational age (weeks) & $25.26 \pm 1.01$ & $25.93 \pm 1.12$ & $25.31 \pm 1.13$ & 0.241 \\
\hline WBC x103/uL & $10.37 \pm 2.47$ & $11.35 \pm 2.45$ & $10.59 \pm 1.99$ & 0.117 \\
\hline $\mathrm{Hgb}(\mathrm{g} / \mathrm{dL})$ & $11.49 \pm 1.13$ & $11.87 \pm 1.05$ & $12.07 \pm 1.06$ & 0.033 \\
\hline Htc $(\%)$ & $34.3 \pm 2.79$ & $35.8 \pm 2.51$ & $36.03 \pm 2.51$ & 0.002 \\
\hline FPG (mg/dL) & - & $80.20 \pm 7.4$ & $89.11 \pm 8.9$ & 0.022 \\
\hline Glucose at $60 \mathrm{~min}(\mathrm{mg} / \mathrm{dL})$ & - & $147.42 \pm 27.27$ & $172.78 \pm 20.79$ & 0.001 \\
\hline Glucose at $120 \mathrm{~min}(\mathrm{mg} / \mathrm{dL})$ & - & $124.66 \pm 22.2$ & $149.50 \pm 14.6$ & 0.001 \\
\hline Glucose at $180 \mathrm{~min}(\mathrm{mg} / \mathrm{dL})$ & - & $113.29 \pm 22.4$ & $130.67 \pm 20.5$ & 0.007 \\
\hline
\end{tabular}

WBC: White blood cell count, Hgb: Hemoglobin, Htc: Hematocrit, FPG: Fasting plasma glucose, $p<0.05$ is accepted as statistically significant. 
132 Yıldırım M. Türkyılmaz E. Demir Cendek B. Dauletkazin G. Alışık M. Baran P. et al.

Table 2: Blood test parameters of each group

\begin{tabular}{|c|c|c|c|c|}
\hline & Control & IGT & GDM & $\mathrm{p}$ \\
\hline \multirow[t]{4}{*}{ Glucose (mg/dL) } & $74.93 \pm 8.56$ & $81.91 \pm 11.87$ & $87 \pm 16.61$ & 0.001 \\
\hline & & & Control-IGT & 0.002 \\
\hline & & & Control-GDM & 0.001 \\
\hline & & & IGT-GDM & 0.379 \\
\hline \multirow[t]{4}{*}{$\mathrm{HgA} 1 \mathrm{c}(\mathrm{g} / \mathrm{dL})$} & $4.92 \pm 0.47$ & $5.25 \pm 0.44$ & $5.74 \pm 0.52$ & 0.001 \\
\hline & & & Control-IGT & 0.001 \\
\hline & & & Control-GDM & 0.001 \\
\hline & & & IGT-GDM & 0.001 \\
\hline \multirow[t]{4}{*}{ Insulin (IU/mL) } & $13.88 \pm 13.81$ & $15.01 \pm 10.28$ & $22.43 \pm 11.99$ & 0.009 \\
\hline & & & Control-IGT & 1.00 \\
\hline & & & Control-GDM & 0.007 \\
\hline & & & IGT-GDM & 0.068 \\
\hline \multirow[t]{4}{*}{ GCT (mg/dL) } & $106.67 \pm 18.64$ & $156.02 \pm 13.12$ & $168.03 \pm 20.26$ & 0.001 \\
\hline & & & Control-IGT & 0.001 \\
\hline & & & Control-GDM & 0.001 \\
\hline & & & IGT-GDM & 0.023 \\
\hline \multirow[t]{4}{*}{ Thiols $(\mu \mathrm{mol} / \mathrm{L})$} & $398.19 \pm 30.49$ & $395.92 \pm 35.52$ & $371.89 \pm 41.14$ & 0.002 \\
\hline & & & Control-IGT & 1.00 \\
\hline & & & Control-GDM & 0.001 \\
\hline & & & IGT-GDM & 0.015 \\
\hline \multirow[t]{4}{*}{ Disulphide $(\mu \mathrm{mol} / \mathrm{L})$} & $17.47 \pm 4.38$ & $19.27 \pm 3.34$ & $25.46 \pm 4.21$ & 0.001 \\
\hline & & & Control-IGT & 0.081 \\
\hline & & & Control-GDM & 0.001 \\
\hline & & & IGT-GDM & 0.001 \\
\hline \multirow[t]{4}{*}{ Disulphide/thiol ratio } & $0.044 \pm 0.012$ & $0.049 \pm 0.009$ & $0.068 \pm 0.0103$ & 0.001 \\
\hline & & & Control-IGT & 0.084 \\
\hline & & & Control-GDM & 0.001 \\
\hline & & & IGT-GDM & 0.001 \\
\hline
\end{tabular}

GCT: Glucose challenge test, HgA1c: Glycosylated hemoglobin A1c, Control-IGT: Comparison of defined parameters between control and IGT groups, Control-GDM: Comparison of defined parameters between control and GDM groups, IGT-GDM: Comparison of defined parameters between IGT and GDM groups. $p<0.05$ is accepted statistically significant

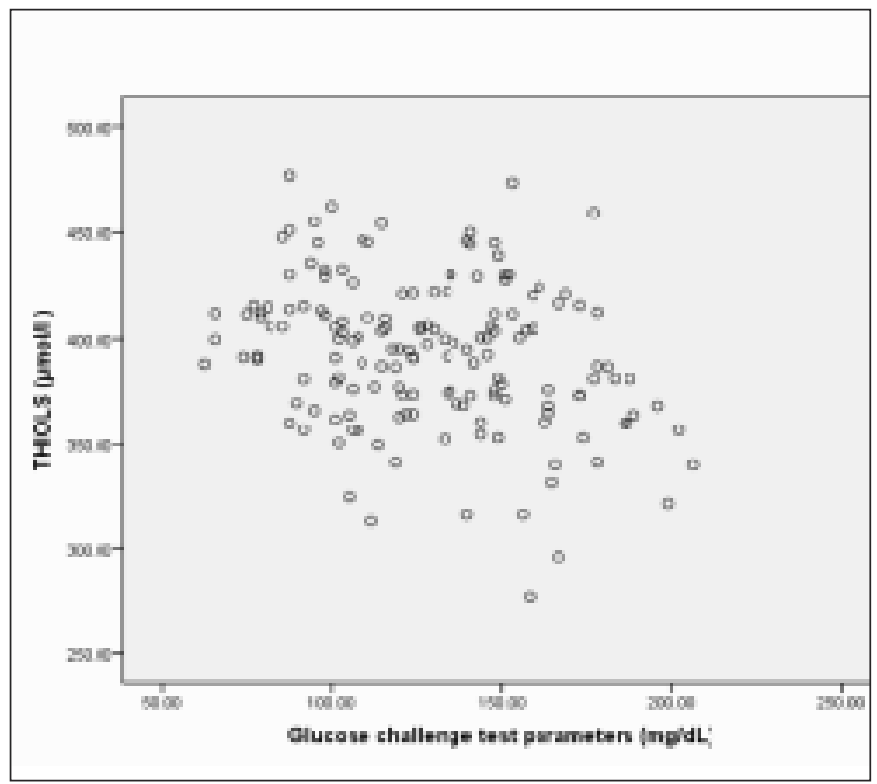

Figure 1: Relationship between maternal serum thiol levels and glucose challenge test values

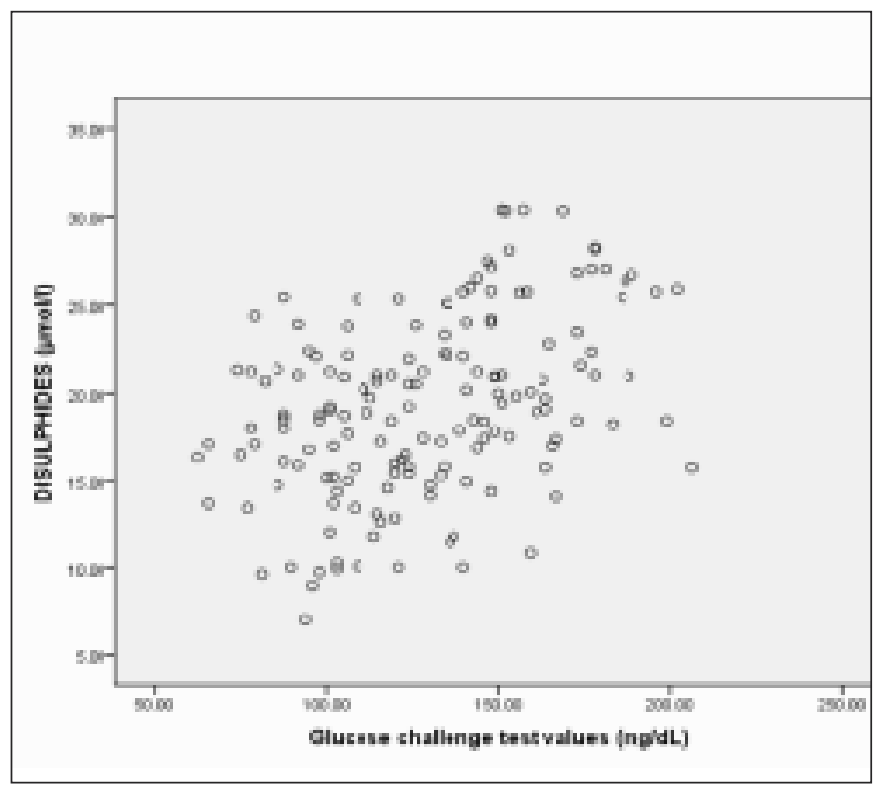

Figure 2: Relationship between maternal serum disulphide levels and glucose challenge test values 


\section{Discussion}

Current study yielded significant findings related to the occurrence of GDM. In normal uncomplicated pregnancies, oxidative stress and lipid peroxidation are relatively increased compared with non-pregnant women (17). However, there is also accompanying increase in antioxidant protection for compensation of increased oxidative stress in these patients(17). In our study, patients with GDM demonstrated elevated levels of disulphide values and reduced thiol levels in their sera comparing to normal healthy pregnancies. Furthermore, dynamic thiol/disulphide homeostasis has shifted in favor of extensive disulphide formation leading to elevation of oxidative stress in GDM patients. Studies pertaining to diabetes and oxidative response have shown that imbalance between ROS and antioxidants may trigger insulin resistance in $\mathrm{DM}(18)$. It was hypothesized that increased oxidative stress as a result of increased production of ROS may damage pancreatic beta cells causing the loss of pancreatic function and finally development of DM (18). Emerging research has shown the possibility of the same mechanism involved in the pathogenesis of GDM as both DM and GDM have a lot in common with respect to development of diseases(19) including hyperglycemia, insulin resistance, impaired insulin secretory response, and hyperlipidaemia.

Limited studies were assessed the relationship between GDM and oxidative stress markers $(20,21)$. Peuchant et al. (10) evaluated oxidant and antioxidant status in pregnant women with either GDM or type 1 DM and they observed reduced antioxidant levels in patients with these conditions compared to the healthy group (10). In another study, plasma and serum maternal total antioxidant and oxidant status were assessed by using different markers in GDM and healthy pregnancies between 24 and 36 weeks of gestation and they found increased oxidative stress and reduced antioxidant levels in patients with GDM (22). They concluded that oxidative stress may contribute to disease processes in this condition (22). However their study population included pregnancies between 24 and 36 weeks of gestation, participants in our study were between 24 and 28 weeks of gestation as being more homogeneous. On the other hand another study found no differences of serum levels of oxidative stress markers between pregnant women with GDM and healthy controls (21). These conflicting results may be due to different criteria chosen by the different research group.

Another study investigated the placental oxidative stress status of women with GDM and they found prominent increase in oxidative stress markers released from placental tissue in the study population (23). They interestingly observed a significant positive correlation between plasma glucose (at 2 hours OGTT) and placental release of oxidative stress markers, however fasting plasma glucose concentrations did not correlate with placental oxidative stress markers (23). This study implies that high blood glucose levels may increase in placental oxidative stress markers leading to the possibility of an adverse effect on the fetus. In our study we failed to demonstrate any relation between thiol/disulphide status and OGTT parameters, however we did observe positive correlation between serum disulphide levels and GCT values and inverse correlation with serum thiol levels and GCT values in the study. Apart from aforementioned studies we also evaluated the antioxidant status in pregnant women with IGT and found significant differences of thiol and disulphide levels between IGT and GDM groups suggesting that oxidative status completely related to blood glucose levels. If blood glucose levels increase, thiol/disulphide homeostasis shifts to the oxidative side in the study. Recent study has shown that women with GDM can develop overt diabetes as late as one decade after pregnancy due to reduced beta-cell reserve as a result of increased oxidative stress during their pregnancies with a sevenfold higher risk than in women without history of GDM (24). Therefore, the correct measurement of oxidants and antioxidants in the serum of pregnant women with GDM become crucial because long term effect of oxidative response in these patients. This study is the first in terms of determining of the levels of thiol/disulphide homeostasis which is more convenient and reliable method identifying oxidative stress in the body (14).

Studies investigating the pathogenesis of obesity have also found close relationship between oxidative stress and the degree of obesity $(25,26)$. Recent study showed that antioxidants especially thiols decreases in the presence of obesity in adults (25). In this current study, all participants were selected from BMI and gestational age-matched pregnant women in order to standardize the study population and prevent the effect of obesity on oxidative stress.

The limitations of the study include the relatively small sample size in terms of GDM group, lack of information regarding the serum antioxidant and oxidant levels in the first trimester and in the third trimester of pregnancies that we might evaluate the effect of oxidative stress on the development and the progress of the disease and the oxidative response to the alteration of blood glucose levels.

In conclusion, based on our finding which decreased antioxidant protection and increased oxidative stress related to increased blood glucose levels were found in patients with GDM, we can infer that blood glucose regulation is extremely crucial for reducing the oxidative stress which may lead to damages to vital organs of a mother or even to development of a fetus in women with GDM. Moreover, it can be considered to adding antioxidant-rich food to diet of particularly diabetic pregnant women with unregulated blood glucose levels may strengthen women immune system against oxidative stress.

\section{References}

1. Metzger BE, Coustan DR. Proceedings of the fourth international workshop-conference on gestational diabetes 
mellitus. Diabetes Care 1998 Aug;21 Suppl 2:B1-167.

2. ADA (American Diabetes Association). Gestational diabetes mellitus position statement. Diabetes Care Diabetes Care 2003 Jan; 26(suppl 1): s103-s105.

3. Gumus II, Kamalak Z, Yuvaci H, Iltemur C, Turhan Ozturk N. Impact of advanced maternal age on perinatal outcomes. Gynecol Obstet Reprod Med 2008;14(1):16-18

4. Whitelaw B, Gayle C. Gestational diabetes. Obstet Gynaecol Reprod Med 2011;21(2):41-46.

5. Halliwell B. Antioxidants and human disease: A general introduction. Nutr Rev 1997;55(1):44-49.

6. Watanabe R, Nakamura H, Masutani H, Yodoi J. Anti-oxidative, anti-cancer and anti-inflammatory actions by thioredoxin 1 and thioredoxin-binding protein-2. Pharmacol Ther 2010;127(3):261-270

7. Brownlee M. Biochemistry and molecular cell biology of diabetic complications. Nature 2001;414(6865):813-820

8. Baynes JW. Role of oxidative stress in development of complications in diabetes. Diabetes 1991;40(4):405-412.

9. West IC. Radicals and oxidative stress in diabetes. Diabet Med 2000;17(3):171-180.

10. Peuchant E, Brun JL, Rigalleau V, Dubourg L, Thomas MJ, Daniel JY. et al. Oxidative and antioxidative status in pregnant women with either gestational or type 1 diabetes. Clin Biochem 2004;37(4):293-298

11. Bayhan G, Bahceci M, Yalinkaya A, Yayla M, Erden AC. Diabetes mellitus in pregnancy and perinatal outcome, Gynecol Obstet Reprod Med 2000;6(2):99-103

12. Go YM, Jones DP. Thiol/disulfide redox states in signaling and sensing. Crit Rev Biochem Mol Biol 2013;48(2): 173-181

13. Circu ML, AwTY. Reactive oxygen species, cellular redox systems, and apoptosis. Free Radic Biol Med 2010;48(6): 749-762.

14. Erel O, Neselioglu S. A novel and automated assay for thiol/disulphide homeostasis. Clin Biochem 2014;47(18):326-32.

15. Carpenter MW, Coustan DR. Criteria for screening tests for gestational diabetes. Am J Obstet Gynecol 1982;144(7):768-773.

16. Marseglia L, Manti S, D’Angelo G, et al. Oxidative stress in obesity: A critical component in human diseases. Int J Mol Sci 2014;16(1):378-400.
17. Walsh SW. Maternal-placental interactions of oxidative stress and antioxidants in preeclampsia. Seminars In Reproductive Endocrinology 1998;16(1):93-104.

18. Ceriello A, Motz E. Is oxidative stress the pathogenic mechanism underlying insulin resistance, diabetes, and cardiovascular disease? The common soil hypothesis revisited. Arterioscler Thromb Vasc Biol 2004;24(5):816823

19. Xiang AH, Peters RK, Trigo E, Kjos SL, Lee WP, Buchanan TA. Multiple metabolic defects during late pregnancy in women at high risk for type 2 diabetes mellitus. Diabetes 1999;48(4):848-854

20. Chaudhari L, Tandon OP, Vaney N, Agarwal N. Lipid peroxidation and antioxidant enzymes in gestational diabetics. Indian J Physiol Pharmacol 2003;47:441-6

21. Orhan H, Önderoglu L, Yücel A, Sahin G. Circulating biomarkers of oxidative stress in complicated pregnancies. Archives of gynecology and obstetrics 2003;267(4):189195.

22. Karacay Ö, Sepici-Dincel A, Karcaaltincaba D, et al. A quantitative evaluation of total antioxidant status and oxidative stress markers in preeclampsia and gestational diabetic patients in 24-36 weeks of gestation. Diabetes Res Clin Prac 2010 Sep;89(3):231-238.

23. Coughlan MT, Vervaart PP, Permezel M, Georgiou HM, Rice GE. Altered placental oxidative stress status in gestational diabetes mellitus. Placenta 2004;25(1):78-84.

24. Bellamy L, Casas JP, Hingorani AD, and Williams D. Type 2 diabetes mellitus after gestational diabetes: a systematic review and meta-analysis. Lancet 2009;373(9677):1773-1779.

25. Vehapoglu A, Turkmen S, Goknar N, Özer ÖF Reduced antioxidant capacity and increased subclinical inflammation markers in prepubescent obese children and their relationship with nutritional markers and metabolic parameters. Redox Rep 2016 Feb 10. (Epub ahead of print)

26. Ottobelli Chielle E, de Souza WM, da Silva TP, Moresco $\mathrm{RN}$, Moretto MB Adipocytokines, inflammatory and oxidative stress markers of clinical relevance altered in young overweight/obese subjects. Clin Biochem 2016;49(7):548-553 